\title{
Quantitative genetic variation in the control of ovarian apoptosis under different environments
}

\author{
M Edvardsson, J Hunt, AJ Moore and PJ Moore \\ Centre for Ecology and Conservation, School of Biosciences, University of Exeter, Penryn, Cornwall, UK
}

\begin{abstract}
Fertility loss in otherwise healthy individuals can be an evolutionary conundrum. Most studies on the evolution of postreproductive lifespan focus on the fitness effects of survival past the age of last reproduction. A complementary approach, which has been largely neglected, is to develop an understanding of the nature of variation in the mechanism underlying loss of fertility, ovarian apoptosis. Variation in the genetics underlying the regulation of ovarian apoptosis could hold the key to understanding the evolution of midlife fertility loss. We estimated quantitative genetic variation in the regulation of ovarian apoptosis in females of the cockroach Nauphoeta cinerea, an insect with reproductive cycles. We have earlier shown that delaying reproduction incites loss of fertility. Here, we forced
\end{abstract}

females to delay reproduction under conditions of excess or limited food and examined apoptosis under both conditions. We found substantial additive genetic variation in levels of apoptosis when females experienced a limited period of starvation during sexual maturation but not when females had unlimited access to food. Hence, selection could act on the regulation of ovarian apoptosis to change the rate of fertility loss with age at least under some environmental circumstances. Our results suggest that an understanding of how loss of fertility evolves requires an understanding of the interaction between genes involved in the regulation of apoptosis and environmental factors such as diet. Heredity (2009) 103, 217-222; doi:10.1038/hdy.2009.44; published online 29 April 2009

Keywords: loss of fertility; oocyte apotosis; quantitative genetics

\section{Introduction}

Female fertility declines with age. In many species this loss of fertility is associated with senescence (Partridge and Fowler, 1992; Kirkwood, 2002). In some species, however, females lose fertility relatively early in their lifespan rather than late in life. This midlife fertility loss can present an evolutionary puzzle; why should otherwise healthy individuals lose reproductive capacity? One possibility is that females have an extended postreproductive lifespan, and contribute to fitness in ways other than personal reproduction. Another possibility is oosorption, the reallocation of reproductive resources to somatic maintenance under conditions of environmental stress. Studies addressing the evolution of loss of fertility have focused on the selective pressures that may be acting on the cessation of reproduction before senescence (Peccei, 2001; Reznick et al., 2006) or on oosorption (Boggs 2009). A complementary approach, which has been largely ignored so far, is developing an understanding of the nature of variation in the target of selection and how this may contribute to its evolution. Here we examine the genetic variation underlying one such target, the control of oocyte apoptosis, the process by which females with a healthy somatic body lose fertility.

Correspondence: Dr PJ Moore, Centre for Ecology and Conservation, School of Biosciences, University of Exeter, Penryn, Cornwall TR10 9EZ, UK.

E-mail:p.j.moore@exeter.ac.uk

Received 11 September 2008; revised 29 January 2009; accepted 19 March 2009; published online 29 April 2009
Ovarian apoptosis has been identified as a universal mechanism of loss of fertility in species ranging from humans (Morita and Tilly, 1999) to insects (Terashima and Bownes, 2004; Moore and Sharma, 2005). Precise control of this evolutionarily conserved mechanism of programmed cell death is essential to the normal functioning of all tissues in all multicellular organisms (Vaux and Strasser, 1996; Reed, 2000; Adams, 2003). Imprecise regulation has disastrous consequences including aberrant embryogenesis, autoimmunity, early loss of fertility and cancer. Thus, we might expect genetic variation in the regulation of apoptosis to be low, as it should be under strong direct selection. However, the decline in midlife fertility is variable (Reznick et al., 2006; Broekmans et al., 2007; Moore et al., 2007) and has the potential to evolve (Moore et al., 2007). This variation may also depend on the specific environmental conditions for an organism. In guppies, for example, the presence of predators results in a delay in the decline in reproductive potential compared with females in populations where predators are absent (Reznick et al., 2006). In humans, phenotypic variation in age at natural menopause also appears to be affected by factors such as smoking and diet (Nagata et al., 2000; Gold et al., 2001; de Bruin et al., 2001; Kok et al., 2005). The variation underlying rates of fertility loss are not only environmental. The age of onset of natural menopause in humans can be predicted by family history, with heritability estimates ranging from 0.30 to 0.85 (Kirk et al., 2001; de Bruin et al., 2001; Kok et al., 2005; Pettay et al., 2005; Broekmans et al., 2007). Linkage analysis of age at natural menopause has identified two significant quantitative trait loci influencing age at menopause 
(van Asselt et al., 2004). Thus, genetic variation does exist in populations in the age at which females lose fertility. The variation in age of onset of post-reproductive lifespan suggests that there must be variation in the loss of oocytes through apoptosis. However, it is unclear where variation might occur in such a highly conserved biochemical pathway.

Age is not the only factor that influences loss of fertility. We expect animals to have evolved mechanisms by which energy saved by a reduction in reproduction can be used to increase survivorship (Bell and Bohm 1975). Oosorption, the ability to resorb eggs, is thought to be an adaptive mechanism by which nutrients can be reallocated into survival when opportunities for reproduction are limited (Bell and Bohm 1975; Papaj 2000; Boggs, 2009). For example, in butterflies a reduction in food leads to a reduction in fecundity, an increase in oocyte resorption, but no reduction in lifespan (Boggs and Ross 1993). This suggests a trade-off between reproduction and lifespan mediated by the recycling of nutrients invested in oocytes that cannot be used. Thus, alleles that may be beneficial in one context, reducing oocyte death and extending relative reproductive lifespan, could be detrimental under other contexts, limiting the ability of individuals to adjust to resource limitation. However, the underlying physiological and genetic control of this trade-off has not been explored. Insight into patterns of resource allocation in varied environments is essential to developing a predictive theory of life history (Boggs 2009), including the relationship between early reproductive history and later life fecundity.

We hypothesize that variation in oocyte and fertility loss arises through variation in the control of apoptosis, through both environmental and genetic factors, rather than in the cell death machinery itself. Thus, a quantitative genetic approach should be informative (Roff, 2007). We tested this hypothesis in the cockroach Nauphoeta cinerea. In $N$. cinerea we can manipulate the rate of fertility loss through both age at first reproduction (Moore and Moore, 2001) and diet (Barrett et al., 2007). $N$. cinerea is ovoviviparous: females carry the embryos in a brood pouch until the offspring are born as first-instar nymphs (Roth, 1964a, b). This species differs from most other invertebrate laboratory systems in that it shows reproductive cycles, with bouts of oocyte maturation followed by pregnancy. Females lose reproductive potential through loss of viable oocytes through apoptosis (Moore and Sharma, 2005) and there is additive genetic variation in fecundity following mating late relative to sexual maturation (Moore et al., 2007).

We investigated the quantitative genetic variation in the levels of ovarian apoptosis in female $N$. cinerea that were forced to delay reproduction by preventing access to males under either ad libitum food or food-restricted conditions by undertaking a paternal half-sib breeding design with broods split between fully fed and foodrestricted conditions during the period between adult eclosion and mating. These treatments were used because diet has been shown to alter rates of apoptosis in N. cinerea (Barrett et al., 2007) and to affect ovarian apoptosis in Drosophila melanogaster; starvation triggers apoptosis in the ovaries resulting in fewer eggs being laid (Terashima and Bownes, 2004). In our study, we found substantial genetic variation in levels of ovarian apoptosis under food-restricted conditions but not under ad libitum food.

\section{Materials and methods}

\section{Animal husbandry}

We obtained the males used as sires and the females used as dams in this study from eight out-bred mixed-sex, mass rearing colonies. The colonies have been established for over 50 years, contain several thousand individuals each, with enforced gene flow among them. The population is maintained under the standard rearing conditions of dry rat chow and water at $28^{\circ} \mathrm{C}$ under a 12:12 h light/dark photoperiod. Assessment of allozyme variation has shown that the population is not inbred (Corley et al., 2001). Last instar nymphs were selected at random from the mass colonies, sexed and placed in single sex colonies in $30 \times 18 \times 12-\mathrm{cm}$ plastic boxes under the standard rearing conditions. When adults emerged they were isolated in individual $11 \times 11 \times 3-\mathrm{cm}$ plastic containers with ad libitum rat chow and water until they were mated. Males were returned to their containers after each mating.

\section{Breeding design and rearing of offspring}

We staged all mating trials under red light in a constant temperature room, kept at $28^{\circ} \mathrm{C}$, during the dark phase of the 12:12 h light/dark photoperiod. Sires $(n=52)$ were mated sequentially to five virgin females each (a minimum of four were included in the analysis), with 3 days $(72 \pm 1 \mathrm{~h})$ between matings. All males were virgin at the time of their first mating and had eclosed as adults 10 days earlier. Dams were also virgin and 10 days posteclosion at the time of mating, the optimal time of mating for females (Moore and Moore, 2001).

We placed mated females in individual $17 \times 12 \times 6.5-$ $\mathrm{cm}$ plastic boxes under the standard rearing conditions. Females were removed from these boxes as soon as the first clutch of offspring appeared. We monitored the boxes and made sure that the developing offspring had access to ad libitum food and water.

\section{Manipulation of experimental females}

We isolated experimental females from each clutch by moving females from the family box into individual $11 \times 11 \times 3-\mathrm{cm}$ plastic boxes on the day they emerged as adults. These females were either provided with ad libitum food and water (three daughters per dam) or were food-restricted, being provided with water only (three daughters per dam). Females were mated at 18 days. Females that mate at sexual maturity (6-9 days post-adult eclosion) have low levels of ovarian apoptosis and high fecundity, whereas females that mate between 9 and 18 days of age show increasing levels of ovarian apoptosis and decreasing lifetime fecundity (Moore and Moore 2001; Moore and Sharma 2005). The reduction in fecundity is seen in both the first clutch produced, but also all clutches produced over a female's lifespan of approximately 300 days.

\section{Apoptosis trials}

After 18 days, we dissected the experimental females and collected 10 mature oocytes from each female. We stained the oocytes for apoptosis using the nucleic acid dye 
YO-PRO-1 and propidium iodide. To confirm that the YO-PRO-1 stain, which is fast and economical (thus allowing for the large numbers of sample necessary for a quantitative genetic study to be stained), represents apoptosis as opposed to other forms of programmed cell death, we stained the oocytes of 18 day post-eclosion females with the stain Casp 3/7 Mito Casp (Bachem, Torrance, CA, USA). This stain is a simultaneous assay for membrane potential and active caspase $3 / 7$. Staining patterns for active caspase $3 / 7$ are identical to those seen with the YO-PRO-1 stain in the ovaries of both ad libitum food and food-restricted 18-day-old females (Figure 1), indicating that YO-PRO-1 does reveal apoptosis in the oocytes under study here. We scored each oocyte depending on the proportion of this epithelium that was stained as follows: (1) $<5 \%$ stained, (2) $5-40 \%$ stained, (3) $40-70 \%$ stained, (4) $70-100 \%$ stained, (5) $100 \%$ stained-the follicular epithelium was completely apoptotic. The score for each female was then calculated by adding the scores of all 10 oocytes.

\section{Statistical methods}

Our experimental design involved a half-sib/full-sib, split brood design with 52 sires and 217 dams. Full-sib families were reared together until the last instar stage, at which time females were separated into individual containers and assigned to either ad libitum food conditions or food-restricted condition. Each family contributed a minimum of two offspring into each environment. Thus, there were 635 individual female offspring assessed for the ad libitum environment and 645 individual offspring for the food-restricted environment.

Our first analysis examined the entire data set with a fully random effects analysis of anova model. Environment was considered a random effect because, similar to family, the specific level of food restriction or abundance was randomly selected from an (nearly) infinite range of possible levels of food. In such a model, the denominator degrees of freedom vary depending on the main effect examined. We used a SAS model III (Ayres and Thomas,
1990; Fry, 1992) where the denominator degrees of freedom for the test of significance for the environment treatment were the sire by environment mean squares; for the test of the effect of sire the denominator mean square was a combination of mean squares (the mean square term for the dam nested within sire + mean square of the sire by environment-mean square for dam nested within sire by environment with 37 degrees of freedom (d.f.)); the test for the dam nested within sire was the dam nested within sire by environment mean squares; the test of the sire by environment effect also used the dam nested within sire by environment means squares; the dam nested within sire by environment effect used the error mean squares in the denominator of the F-test.

Within environments, we calculated quantitative genetic parameters from our half-sib/full-sib design using restricted maximum likelihood method to generate variance estimates. The level of imbalance in our data was minor, with little difference between restricted maximum likelihood and least square estimates, and we therefore calculate significance from least-squares estimates of expected means squares in a nested analysis of variance (Lynch and Walsh, 1998).

\section{Results}

The levels of ooctye apoptosis differed under the two environmental conditions (Figure 2). Controlling for genetic influences by analyzing the data with a fully random effects analysis of variance model (with environment, sire, dam nested under sire and their interactions), there was a highly significant difference in the apoptosis scores in the ad libitum food environment compared with the food-restricted environment (Table 1). The average apoptosis score in the ad libitum environment was 12.8 (s.d.=3.2), whereas the average apoptosis score in the food-restricted environment was 18.3 $(\mathrm{s} . \mathrm{d} .=12.0)$. Although there was no significant effect of sire or dam in the overall model, both terms significantly
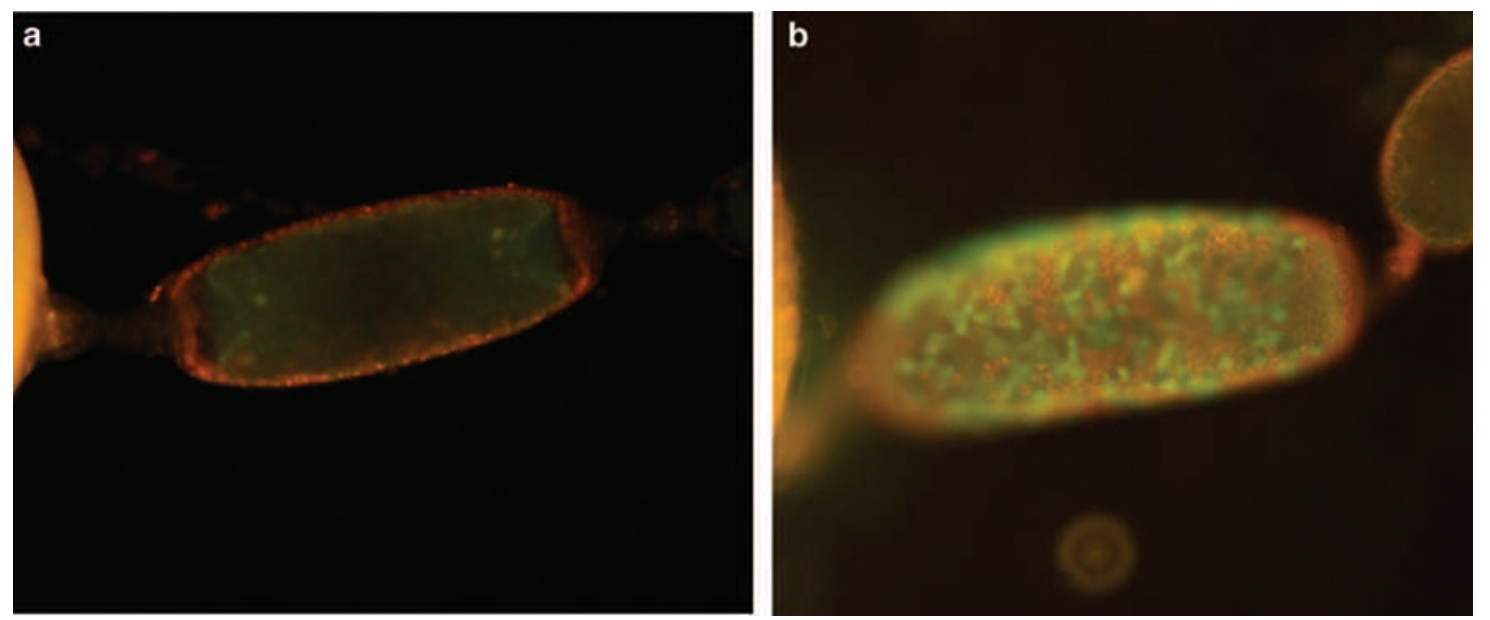

Figure 1 Apoptosis occurs in the ovaries of females that delay mating relative to sexual maturation. Ovaries from ad libitum food females 18 days post-adult eclosion are stained with a simultaneous assay for membrane potential (red fluorescence; Casp $3 / 7$ Mito Casp, Bachem) and active caspase (green fluorescence; carbocyfluorescein-labeled fluoromethyl ketone peptide inhibitor). Apoptosis occurs within oocytes (a; apoptotic oocyte surrounded by healthy follicle cells) and also in the follicle cells that act as nurse cells to the developing oocytes (b; a mixture of healthy and apoptotic follicle cells surrounding the immature oocyte). Similar staining patterns are seen in ovaries of foodrestricted females. 


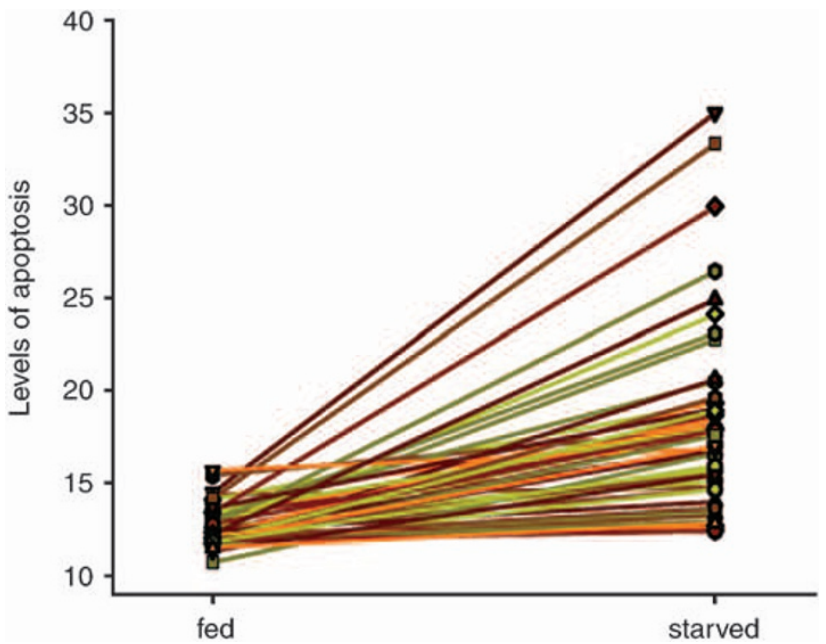

Figure 2 The levels of apoptosis expressed as sire family means of scores from mature oocytes of 18-day-old females. Each line connects the mean score of the daughters of one sire in the two treatments. The level of apoptosis was scored in 10 mature oocytes from a female offspring of each dam and the mean was then calculated for each dam.

Table 1 Partially nested two-way analysis of variance from SAS GLM investigating factors that influence oocyte apoptosis in N. cinerea

\begin{tabular}{lrrrc}
\hline Factor & d.f. & \multicolumn{1}{c}{ MS } & F-value & P-value \\
\hline Food level & 1 & 9132.07 & 66.71 & $<0.001$ \\
Sire & 51 & 188.43 & 1.43 & 0.13 \\
Dam (sire) & 165 & 89.17 & 0.94 & 0.66 \\
Sire $\times$ food level & 51 & 137.62 & 1.45 & 0.04 \\
Dam (sire) $\times$ food level & 165 & 95.18 & 1.56 & $<0.001$ \\
Error & 846 & 60.91 & & \\
\hline
\end{tabular}

Table 2 Separate REML estimates of components of variation underlying oocyte apoptosis for females reared under two conditions

\begin{tabular}{llllllll}
\hline Treatment & $\mathrm{V}_{A}$ & $\mathrm{~V}_{C}$ & $\mathrm{~V}_{E}$ & $\mathrm{~V}_{P}$ & $h^{2} \pm$ s.e. & $\mathrm{N}_{\text {sire }}$ & $\mathrm{N}_{\text {dam }}$ \\
$\mathrm{N}_{\text {offsp }}$
\end{tabular}

Ad libitum food $0.00492 .12 \quad 8.00 \quad 10.120 .00 \pm 0.11 \quad 52 \quad 218 \quad 636$

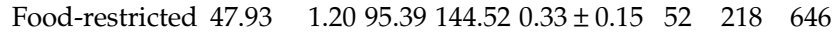

$V_{\mathrm{A}}$ is the additive genetic variance; $V_{\mathrm{C}}$ is the variance attributable to dominance, epistasis and common environment; $V_{\mathrm{E}}$ is the environmental variance; $V_{\mathrm{P}}$ is the phenotypic variance; $\mathrm{h}^{2}$ is the narrow sense heritability.

interacted with the environment (Table 1). Thus, the expression and control of apoptosis in the two environments were clearly different.

Our design allowed us to estimate genetic influences on the levels of apoptosis within each environment (Table 2). In the ad libitum food environment, there was no significant effect of sire $(F=0.93$, d.f. $=51,165$, $P=0.62)$ but a significant effect of dam nested within sire $(F=1.65$, d.f. $=165,418, P<0.001)$, In contrast, in the food-restricted environment we found both a significant sire effect $(F=1.82$, d.f. $=51,165, P=0.002)$ and a significant dam nested within sire effect $(F=1.54$, d.f. $=165,428, P<0.001)$, In food-restricted females, the environmental variance increased relative to that seen in fed females (Table 2, $V_{\mathrm{E}}$ ), as would be expected under stressful conditions (Hoffmann and Parsons, 1991), but this increase alone did not explain the increase of phenotypic variance observed. Both conditions exhibited significant non-additive and common environment effects (Table 2, $V_{C}$ ) but there was relatively little difference in the non-additive genetic and common environment variance contribution between fed and food-restricted females. In contrast, in the ad libitum food environment, there was a lack of additive genetic variation compared with a significant level of additive genetic variation in the food-restricted environment (Table 2, $V_{\mathrm{A}}$ ). Over $30 \%$ of the variation in levels of apoptosis observed in the ovaries of females under nutritional stress during sexual maturation can be attributed to additive genetic differences among families, whereas this is nearly zero in the ad-libitum food environment.

\section{Discussion}

We have provided the first demonstration of quantitative genetic variation in the levels of ovarian apoptosis and, indeed, of the regulation of apoptosis in any tissue. Our results show that ovarian apoptosis is turned on in response to nutritional stress, but to what extent depends on the genotype of the individual female. Therefore, in our population of cockroaches, selection could act on the regulation of ovarian apoptosis to change the rate of fertility loss with age under some environmental circumstances.

Although no other studies have examined quantitative genetic variation in the control of apoptosis, there is reason to believe that the complex interaction between environment and genetic variation we have uncovered in $N$. cinerea mirrors the control of ovarian apoptosis in other species. Ovarian apoptosis is involved in maintaining optimal functioning of the mammalian ovary (Amsterdam et al., 1999), where fertility is lost with age through the loss of ovarian follicles through apoptosis (Morita and Tilly, 1999). Female mice in which the gene for Bax, a key regulator of apoptosis, has been knocked out have a prolonged ovarian lifespan (Perez et al., 1999). In addition, in a linkage analysis of age at menopause, one of the two quantitative trait loci that explained variation in age of onset of menopause in humans was a region on chromosome 9 that contains a gene from the BCL2 family, an important protein in the apoptotic pathway (van Asselt et al., 2004). Therefore, the potential exists for similar genetic variation in control of apoptosis over time in humans.

Apoptosis is critical to a large number of processes, which may mean that the genes controlling apoptosis often are under conflicting selection pressures and face trade-offs between different functions. However, our results highlight the fact that natural selection is unlikely to have removed all allelic variation in control of apoptosis for every function, be it apoptosis of oocytes or suppression of cancer, under all environmental circumstances. It is well documented that the heritability of a trait can change under different environmental conditions (Sgrò and Hoffmann, 1998; Hoffmann and Merilä, 1999). Heritability can decrease under stress due to an increased environmental variance or change because the history of selection differs between environ- 
ments (Hoffmann and Parsons, 1991). Selection in a stable environment should decrease additive genetic variance, as alleles with favorable effects become fixed in the population. More additive genetic variance may remain for the expression of traits in environments that are encountered only rarely and where selection is therefore expected to be relatively weak.

In this study, the heritability of levels of apoptosis increased under nutritional stress because of additive genetic contributions to the phenotypic variation. Ovarian apoptosis in females that delay reproduction under conditions of adequate nutritional resource may reflect a developmental pathway to remove oocytes from the ovary at the end of an unproductive reproductive cycle to clear the system for the next cycle (Tyndale-Biscoe and Watson, 1977). The lack of additive genetic variance in this trait under benign conditions indicates that there has been strong directional or stabilizing selection on the levels of apoptosis in the oocytes as females pass sexual maturation without obtaining a mate. The induction of apoptosis in the ovaries of food-restricted females, however, is not simply a developmental response. These females are also responding to the environmental insult of nutritional stress. The observed increase in the additive genetic variance reflects the contribution of genes that control the onset of apoptosis only under nutritional stress. Because families were split between environments, we can show that the increase in additive genetic variance occurs even within families. However, because there is no heritability within one of the environments, this is not a genotype by environment interaction in which we would observe the change in expression of one set of genes over two environments. Rather, what we have measured is the action of genes controlling the initiation of the apoptotic pathway under nutritional stress, but not when females are fully fed. These genes are under different selection than the selection experienced by the genes controlling apoptosis during normal developmental progression. Interestingly, females that mate late relative to sexual maturation in the absence of food have higher fecundity and increased longevity compared with females that delay mating in the presence of food (Barrett et al., 2009). Thus, the difference in genetic architecture of ovarian apoptosis in starved females that delay mating is reflected in variation in fitness consequences of timing of reproduction in the two nutritional environments.

The role of nutritional stress on ovarian function and apoptosis has been best studied in D. melanogaster. Starved female $D$. melanogaster have significantly fewer egg chambers at stage $8 / 9$ due to an increase in apoptosis (Terashima and Bownes, 2004). Broad-Complex (BR-C) is a key gene for selecting between apoptosis and oocyte development, with differential expression of BR-C isoforms under nutritional stress, leading to the induction of apoptosis rather than yolk protein synthesis and oocyte development. Individual variation in expression of the BR-C isoforms has not been examined, but it is likely that such variation exists. Substantial genetic variation in starvation resistance in the natural populations has been documented (Harbison et al., 2004) and an increase in starvation resistance is often negatively correlated with early fecundity (Service and Rose, 1985). The physiological mechanisms underlying the mediation of trade-offs between starvation resistance and fecundity are largely unknown (Wayne et al., 2006). Apoptosis of oocytes, however, would seem to play an important role in this process.

The evolution of ovarian apoptosis is complex. A full understanding of why reproduction may end early in a female's lifespan requires an integration of how genetics and selection might influence the physiological mechanisms that result in a loss of fertility, as well as these influences in various environments, including variation in access to resources. The results of this study provide insight into the complexity of the individual variation upon which selection could be acting, and this additional complexity needs to be incorporated into our considerations of the evolution of fertility loss.

\section{Acknowledgements}

We thank F Champion de Crespigny for practical help during the experiment. F Champion de Crespigny, D Hosken and $\mathrm{T}$ Tregenza provided constructive comments on an earlier draft of this paper. This research was supported by a Leverhulme grant to PJM and AJM. JH was supported by a National Environmental Research Council Fellowship.

\section{References}

Adams JM (2003). Ways of dying: multiple pathways to apoptosis. Genes Dev 17: 2481-2495.

Amsterdam A, Gold RS, Hosokawa K, Yoshida Y, Sasson R, Jung YS, Kotsuji F (1999). Crosstalk among multiple signaling pathways controlling ovarian cell death. Trends Endocrin Met 10: $255-262$.

Ayres MP, Thomas DL (1990). Alternative formulations of the mixed-model ANOVA applied to quantitative genetics. Evolution 44: 221-226.

Barrett ELB, Moore AJ, Moore PJ (2009). Diet and social conditions during sexual maturation have unpredictable influences on female life history trade-offs. J Evol Biol 22: 571-581.

Barrett ELB, Preziosi RF, Moore AJ, Moore PJ (2007). Effects of mating delay and nutritional signals on resource recycling in a cyclically breeding cockroach. I Insect Physiol 54: 25-31.

Bell WJ, Bohm MK (1975). Oosorption in insects. Biol Rev 50: 373-396.

Boggs CL (2009). Understanding insect life histories and senescence through a resource allocation lens. Func Ecol 23: 27-37.

Boggs CL, Ross CL (1993). The effect of adult food limitation on life history traits in Speyeria mormonia (Lepidoptera: Nymphalidae). Ecology 74: 433-441.

Broekmans FJ, Knauff EAH, te Velde ER, Macklon NS, Fauser BC (2007). Female reproductive ageing: current knowledge and future trends. Trends Endocrin Met 18: 58-65.

Corley LS, Blankenship JR, Moore AJ (2001). Genetic variation and asexual reproduction in the facultatively parthenogenetic cockroach Nauphoeta cinerea: implications for the evolution of sex. J Evol Biol 14: 68-74.

de Bruin JP, Bovenhuis $\mathrm{H}$, van Noord PAH, Pearson PL, van Arendonk JAM, Veldt ERT, Kuurman WW, Dorland M (2001). The role of genetic factors in age at natural menopause. Hum Reprod 16: 2014-2018.

Fry JD (1992). The mixed-model analysis of variance applied to quantitative genetics: biological meaning of the parameters. Evolution 46: 540-550.

Gold EB, Bromberger J, Crawford S, Samuels S, Greendale GA, Harlow SD, Skurnick J (2001). Factors associated with age at natural menopause in a multiethnic sample of midlife women. Am J Epidemiol 153: 865-874. 
Harbison ST, Yamamoto AH, Fanara JJ, Norga KK, Mackay TFC (2004). Quantitative trait loci affecting starvation resistance in Drosophila melanogaster. Genetics 166: 1807-1823.

Hoffmann AA, Merilä J (1999). Heritable variation and evolution under favourable and unfavourable conditions. Trends Ecol Evol 14: 96-101.

Hoffmann AA, Parsons PA (1991). Evolutionary Genetics and Environmental Stress. Oxford University Press: Oxford. pp 97-142.

Kirk KM, Blomberg SP, Duffy DL, Heath AC, Owens IPF, Martin NG (2001). Natural selection and quantitative genetics of life-history traits in western women: a twin study. Evolution 55: 423-435.

Kirkwood TBL (2002). Evolution of ageing. Mech Ageing Dev 123: $737-745$.

Kok HS, van Asselt KM, van der Schouw YT, Peeters PHM, Wijmenga C (2005). Genetic studies to identify genes underlying menopausal age. Hum Reprod Update 11: 483-493.

Lynch M, Walsh B (1998). Genetics and Analysis of Quantitative Traits. Sinauer Associates: Sunderland.

Moore PJ, Harris WE, Moore A J (2007). The cost of keeping eggs fresh: quantitative genetic variation in females that mate late relative to sexual maturation. Am Nat 169: 311-322.

Moore PJ, Moore AJ (2001). Reproductive aging and mating: the ticking of the biological clock in female cockroaches. Proc Natl Acad Sci USA 98: 9171-9176.

Moore PJ, Sharma SA (2005). A delay in age at first mating results in the loss of future reproductive potential via apoptosis. Evol Dev 7: 216-222.

Morita Y, Tilly JL (1999). Oocyte apoptosis: like sand through an hourglass. Dev Biol 213: 1-17.

Nagata C, Takatsuka N, Kawakami N, Shimizu H (2000). Association of diet with the onset of menopause in Japanese women. Am I Epidemiol 152: 863-867.

Papaj DR (2000). Ovarian dynamics and host use. Ann Rev Entomol 45: 423-448.

Partridge L, Fowler K (1992). Direct and correlated responses to selection on age at reproduction in Drosophila melanogaster. Evolution 46: 76-91.

Peccei JS (2001). Menopause: adaptation or epiphenomenon? Evol Anthropol 10: 43-57.
Perez GI, Robles R, Knudson CM, Flaws JA, Korsmeyer SJ, Tilly JL (1999). Prolongation of ovarian lifespan into advanced chronological age by Bax-deficiency. Nat Genet 21: 200-203.

Pettay JE, Kruuk LEB, Jokela J, Lummaa V (2005). Heritability and genetic constraints of life-history trait evolution in preindustrial humans. Proc Natl Acad Sci USA 102: 2838-2843.

Reed JC (200). Mechanisms of apoptosis. Am J Pathol 157: $1415-1430$

Reznick D, Bryant M, Holmes D (2006). The evolution of senescence and post-reproductive lifespan in guppies (Poecilia reticulata). PLoS Biology 4: 0136-0143.

Roff DA (2007). A centennial celebration for quantitative genetics. Evolution 61: 1017-1032.

Roth LM (1964a). Control of reproduction in female cockroaches with special reference to Nauphoeta cinerea. I. First pre-oviposition period. J Insect Physiol 10: 915-945.

Roth LM (1964b). Control of reproduction in female cockroaches with special reference to Nauphoeta cinerea. II. Gestation and postparturition. Psyche 71: 198-244.

Service PM, Rose MR (1985). Genetic covariation among lifehistory components: the effect of novel environments. Evolution 39: 943-945.

Sgrò CM, Hoffmann AA (1998). Effects of stress combinations on the expression of additive genetic variation for fecundity in Drosophila melanogaster. Genetical Research 72: 13-18.

Terashima J, Bownes M (2004). Translating available food into the number of eggs laid by Drosophila melanogaster. Genetics 167: 1711-1719.

Tyndale-Biscoe M, Watson JAL (1977). Extra-ovariolar egg resorption in a dung beetle, Euoniticellus intermedius. J Insect Physiol 23: 1163-1167.

van Asselt KM, Kok HS, Putter H, Wijmenga C, Peeters PHM, van der Schouw YT, Grobbee DE, te Velde ER, Mosselman S, Pearson PL (2004). Linkage analysis of extremely discordant and concordant sibling pairs identifies quantitative trait loci influencing variation in human menopausal age. Am J Hum Genet 74: 444-453.

Vaux DL, Strasser A (1996). The molecular biology of apoptosis. Proc Natl Acad Sci USA 93: 2239-2244.

Wayne ML, Soundararajan U, Harshman LG (2006). Environmental stress and reproduction in Drosophila melanogaster: starvation resistance, ovariole numbers and early age egg production. BMC Evol Biol 6: 57-66. 